\title{
Molecular cloning and expression of GPX gene from Siniperca chuatsi
}

\author{
Rui-hong Ning ${ }^{1}$, Ming Zhang ${ }^{2}$, Zhou-yu Chen ${ }^{1}$, Bao-qing $\mathrm{Hu}^{1}$, Chun-gen Wen ${ }^{1 *}$
}

1. School of Life Sciences, Nanchang University, Nanchang 330031, China

2. College of Jiangxi Biotech Vocational, Nanchang 330200, China

\begin{abstract}
Glutathione peroxidases are one of the main enzymes in antioxidant defence system of the living organisms. In the present study, an atypical GPx gene designated as HcGPx, was identified from Siniperca chuatsi. The full-length cDNAs gene of GPX was $948 \mathrm{bp}$, including a complete protein coding region of 564bp, a 3'untranslated region of $384 \mathrm{bp}$. A selenocysteine (Sec) was encoded by TGA of an unusual stop codon, with the selenocysteine insertion sequence ( SECIS) element located at 3' UTR. The SECIS element from $S$. chuatsi was compared to ones from other fishes, which was high conservative and was belonged to from1. Structure of a deduced amino acid sequence showed that GPX of $S$. chuatsi had Gln71 and Trp148 of Sec catalytic sites, three loops of stable enzyme tertiary structure, PGGG motif, and homotetramer consisted of selenocysteine, tryptophan, glutamine and asparagine. Comparison GPX of $S$. chuatsi with one of other vertebrate animals revealed that the similarity of amino acid sequence was $66 \%-91 \%$. Phylogenic analysis indicated that three independent clades were apparent, GPX1、GPX2 and GPX3 located in the same clade, whereas GPX4 belonged to a different clade. Real-time quantitative PCR was used to analyze the GPX gene expression characterization in tissues of $S$. chuatsi. The results showed that expression of GPX gene was found in all detected tissues, and expression level was the highest in the tissues of head kidney, liver, gill, and had significant differences in in different tissues.
\end{abstract}

Key words: Siniperca chuatsi; glutathione peroxidase; Gene cloning; expression.

\footnotetext{
* Corresponding author: Tel.: +86-0791-3969530; fax: +86-0791-3969530. E-mail address: cgwen@ncu.edu.cn (CG, Wen)
} 